\title{
Composición corporal y variabilidad de la frecuencia cardiaca: relaciones con edad, sexo, obesidad y actividad física
}

\author{
Body composition and heart rate variability: \\ relations to age, sex, obesity and physical activity
}

López Sánchez, G.F., López Sánchez, L. y Díaz Suárez, A.

Facultad de Ciencias del Deporte, Universidad de Murcia, España

Resumen: La composición corporal (CC) y la variabilidad de la frecuencia cardiaca (VFC) están estrechamente relacionadas con la salud de las personas. Las dos variables han sido estudiadas en numerosas ocasiones por separado, sin embargo, son pocas las investigaciones que han abordado en conjunto las relaciones entre los dos conceptos, ofreciendo en muchos casos, resultados contradictorios. El principal objetivo de este trabajo es realizar una revisión de la literatura científica que trata la relación entre la CC y la VFC desde distintos puntos de vista: en relación a la edad (niños y adolescentes, adultos y mayores), en relación a la obesidad, al sexo y a la cantidad de actividad física realizada. Esta revisión, permite concluir que existe una relación entre la CC y la VFC, de tal forma que una disminuida VFC está asociada a un alto porcentaje de grasa corporal y a un menor porcentaje de masa muscular. Palabras clave: Composición Corporal, Frecuencia Cardiaca, Salud, Obesidad, Actividad Física.
Abstract: Body composition (BC) and heart rate variability (HRV) are closely related to the health of people. The two variables have been studied separately on numerous occasions, however, few studies have studied the whole relationship between the two concepts with, in many cases, conflicting results. The main objective of this paper is to review the scientific literature dealing with the relationship between BC and HRV from different points of view: in relation to age (children and adolescents, adults and seniors) in relation to obesity, sex and amount of physical activity. This review leads to the conclusion that there is a relationship between $\mathrm{BC}$ and HRV, so that decreased HRV is associated with a high percentage of body fat and a lower percentage of muscle mass.

Keywords: Body Composition, Heart Rate, Health, Obesity, Physical Activity.

\section{Justificación}

La composición corporal (CC) es un concepto ampliamente estudiado, y que ha demostrado ser uno de los parámetros que más estrechamente se relacionan con el estado nutricional, así como, con la salud de las personas (Alvero et al., 2010). Existen numerosos trabajos que consideran que el análisis de la composición corporal permite comprender el efecto que tienen la dieta, el crecimiento, la actividad física, la enfermedad y otros factores del entorno sobre las proporciones de los distintos constituyentes del cuerpo humano y su estudio, por tanto, resulta imprescindible (Björntorp, 1992; Moreno, 2000; Grund et al., 2001; Oria et al, 2002; Christou et al., 2004; Alburquerque, 2008). Es por ello, que la composición corporal constituye el eje central de la valoración del estado nutricional, de la monitorización de pacientes con malnutrición aguda o crónica, y del diagnóstico y tipificación del riesgo asociado a la obesidad (Moreno, 2000).

Por otro lado, la variabilidad de la frecuencia cardiaca $(\boldsymbol{V F C})$ es el término que describe las variaciones de la frecuencia cardiaca instantánea y del intervalo de tiempo que transcurre entre cada latido, conocido comúnmente como

Dirección para correspondencia [Correspodence address]: Guillermo Felipe López Sánchez. Facultad de Ciencias del Deporte, Universidad de Murcia (España).E-mail: gfls@um.es intervalo RR (Grupo de Trabajo de la Sociedad Europea de Cardiología y la Sociedad Norte Americana de Electrofisiología, 1996). Autores más recientes, como Rodas et al. (2008), la han definido como la variación de la frecuencia del latido cardiaco durante un intervalo de tiempo definido.

Es bien sabido, que la variabilidad de la frecuencia cardiaca está directamente relacionada con el control que el sistema nervioso autónomo ejerce sobre los aparatos cardiovascular y respiratorio (Maud \& Foster, 1991; Gallo et al., 1999; Pumprla et al., 2002; Kleiger et al., 2005). Por lo tanto, su determinación nos puede aportar información muy útil y valiosa sobre el estado de salud, así como sobre las adaptaciones que se producen a causa del entrenamiento.

En este sentido, la VFC puede aportar información relacionada con el pronóstico, patogénesis y estrategias de tratamiento en pacientes con patología cardiovascular (Kop et al., 2001), progreso de la diabetes mellitus (Malpas \& Maling, 1990), control del estado de fibromialgia (Martinez-Lavin, 2002) y síndrome de fatiga crónica (De Becker et al., 1998).

Además, la VFC ha sido utilizada para estudiar los estados de adaptación al estrés físico (Hall et al., 2004) y psíquico (Sibolboro et al., 2001). Actualmente, su análisis nos aporta información relativa a las adaptaciones producidas, tanto por el entrenamiento como durante la competición, pudiendo 
ser un buen marcador predictivo de estados de sobreentrenamiento (Hynynen et al., 2006; Achten \& Jeukendrup, 2003).

Tanto la variabilidad de la frecuencia cardiaca como la composición corporal, han sido estudiadas en numerosas ocasiones por separado, sin embargo, son pocas las investigaciones hasta el momento que aborden en conjunto las relaciones entre estos dos conceptos. Uno de los primeros estudios sobre el tema fue el de Zahorska-Markiewicz et al. (1993) que estudió la relación entre obesidad y variabilidad de la frecuencia cardiaca. Desde entonces, cabe destacar varios estudios que han relacionado la variabilidad de la frecuencia cardiaca con la masa grasa (Christou et al., 2004; Esco et al., 2011; Kim et al., 2005; Martínez et al., 2011), la masa muscular (Martínez et al., 2011) y el tamaño corporal (Freeman et al., 1995; Molfino et al., 2009) en adultos sanos. En niños y adolescentes se ha puesto de manifiesto que las mayores relaciones se producen entre la masa grasa y la VFC (Gutin et al., 1997; Kaufman et al., 2007a; Kaufman et al., 2007b; Martini et al., 2001; Gutin et al., 2005; Millis et al., 2010).

No obstante, hasta la fecha, las diferentes investigaciones que han tratado este tema de forma directa o indirecta ofrecen, en muchos casos, resultados contradictorios. Debido a la estrecha relación que estas dos variables guardan con la salud, parece pertinente la realización de una revisión profunda de la literatura, a fin de organizar y aclarar el conocimiento científico más relevante sobre este tema.

Por lo tanto, como principal objetivo de este trabajo se propone ordenar y sentar las bases sobre las cuales podrán apoyarse futuros trabajos que estudien de forma experimental la relación existente entre la composición corporal y la variabilidad de la frecuencia cardiaca.

\section{Criterios de búsqueda}

Para la realización de este trabajo, se han utilizado las principales herramientas para búsquedas de información científica, a las cuales se puede acceder a través de internet. Las fuentes de información consultadas han sido las siguientes:

$\checkmark$ Medline: Base de datos de bibliografía médica producida por la Biblioteca Nacional de Medicina de los Estados Unidos (http://www.ncbi.nlm.nih.gov/pubmed/).

$\checkmark$ Web of Knowledge (WOK): Colección de bases de datos bibliográficas, de citas y referencias de publicaciones de carácter científico y tecnológico (http://www.accesowok.fecyt.es/).

$\checkmark$ ScienceDirect: Colección electrónica de textos de tecnología, medicina y de información bibliográfica (http:// www.sciencedirect.com/).

$\checkmark$ RECOLECTA o Recolector de Ciencia Abierta: Plataforma que agrupa a todos los repositorios científicos nacionales (http://www.recolecta.net/buscador/).
La búsqueda principal se ha realizado introduciendo los siguientes descriptores: "body composition", "body composition measurement", "body composition and health", "heart rate variability", "heart rate variability measurement", "heart rate variability and health", "body composition and heart rate variability".

Los mismos descriptores, pero en español, han sido utilizados para realizar la búsqueda en bases de datos que permiten el acceso a publicaciones cuyo lenguaje es el español: "composición corporal", "medición de la composición corporal", "composición corporal y salud", "variabilidad de la frecuencia cardiaca", "medición de la variabilidad de la frecuencia cardiaca", "variabilidad de la frecuencia cardiaca y salud", "composición corporal y variabilidad de la frecuencia cardiaca".

Es importante remarcar el hecho de que se ha incluido, únicamente, información proveniente de artículos de revistas científicas, tesis doctorales, actas de congresos científicos y libros de fisiología básica y aplicada. Se han excluido de la revisión otro tipo de publicaciones, tales como tesis de máster o trabajos fin de máster, noticias de prensa o información contenida en "blogs" de internet.

\section{Sobre la relación entre la CC y la VFC}

Aunque no es abundante, existe literatura que trata la relación entre la CC y la VFC desde distintos puntos de vista. Por ello, se ha decidido presentar las investigaciones más relevantes al respecto en relación a la edad (niños y adolescentes, adultos y mayores), en relación a la obesidad, al sexo y a la cantidad de actividad física realizada.

\subsection{En relación a la edad.}

En niños y adolescentes, los perfiles más favorables de VFC se asocian con más actividad física moderada-vigorosa, con mejor condición física cardiovascular y con menos adiposidad visceral y subcutánea. Por ello la mejora de la modulación cardiaca autónoma puede ser una vía a través de la cual la actividad física, la condición física y la delgadez contribuyen a la salud cardiovascular desde edades tempranas (Gutin et al., 2005). En esta línea, se ha observado que el porcentaje de grasa corporal se correlaciona negativamente con el cociente baja frecuencia/alta frecuencia $(\mathrm{LF} / \mathrm{HF})$ durante la respiración no controlada pero no durante la respiración rítmica. Además, un alto porcentaje de grasa corporal puede estar asociado con una baja modulación simpática de la FC en adolescentes/jóvenes sanos de sexo masculino (Millis et al., 2010).

En el caso de los adultos y mayores, Freeman et al. (1995) señalaron que el peso y el IMC son predictores significativos del cociente expiración/inspiración (E/I ratio) y de la diferencia entre la FC máxima y mínima (HRMax-Min), lo que indica que la VFC y el tamańo corporal están correlaciona- 
dos. Por tanto, una disminución de la VFC, mediada por el SNP, podría explicar en parte la mortalidad y la morbilidad asociadas con la obesidad.

En esta línea, se ha observado una asociación negativa entre la RMSSD (que refleja la VFC) y LF (que refleja la actividad simpática) con el porcentaje de masa grasa y con el ratio cintura/cadera en personas obesas, lo que indica que la obesidad puede cambiar la respuesta cardiaca nerviosa autónoma, es decir, que el mecanismo por el cual la obesidad aumenta la mortalidad cardiaca sería explicado, al menos parcialmente (Kim et al., 2005). Tanto una mayor distribución de grasa corporal abdominal-periférica, como una mayor adiposidad abdominal o una mayor adiposidad total se asocian con menores expresiones de VFC (Christou et al., 2004). No obstante, algunos autores, como Fagard et al. (1999), señalan que los efectos del tamaño corporal y el IMC en la VFC son escasos e insignificantes.

La modulación cardiovascular autónoma está relacionada significativamente con la capacidad aeróbica máxima y con la composición corporal, siendo el sumatorio de pliegues cutáneos (pecho, abdomen y muslo) el parámetro que tiene la correlación independiente más fuerte con la VFC y con la recuperación de la FC (Esco et al., 2011). Un aumentado tono simpático (LF) y VLF (relacionado con la termogénesis) en hombres sanos se relaciona con un mayor porcentaje de músculo esquelético, y un aumentado tono vagal (HF) y tono parasimpático (LF/ HF) en mujeres sanas, se relaciona con un aumento del porcentaje de grasa corporal (Martinez et al., 2011).

Según Molfino et al. (2009), las HF (índice de la modulación parasimpática) en individuos con un IMC $<20 \mathrm{~kg} / \mathrm{m}^{2}$ son significativamente más altas que las HF de los demás sujetos. Estos resultados apoyan el rol de cómo la actividad parasimpática ejerce una influencia sobre el IMC a través de una probable modulación del peso corporal. Por último, en algunos estudios se han observado alteraciones del control simpático vagal de la VFC en personas con desórdenes alimenticios, aunque estos cambios no parecen estar relacionados con el peso corporal y el IMC (Murialdo et al., 2007).

A continuación se presentan cuatro tablas, que recogen y resumen los trabajos más destacados que han estudiado la relación entre la CC y la VFC en función de la edad, así como las relaciones más importantes encontradas por los diversos autores entre estos dos conceptos. Analizando las mismas, se puede observar las importantes implicaciones que estos resultados tienen en relación al estado de salud, ya que unos valores más saludables de CC, que a su vez se asocian a una mejor condición física, parecen influir positivamente en la VFC de las personas.

Tabla 1. Estudios destacados sobre la relación CC-VFC en niños y adolescentes.

\begin{tabular}{|c|c|c|c|c|}
\hline Referencia & Muestra & $\begin{array}{l}\text { Medida } \\
\text { de la CC }\end{array}$ & $\begin{array}{l}\text { Medida de } \\
\text { la VFC }\end{array}$ & Relación CC/VFC \\
\hline $\begin{array}{l}\text { Gutin et al. } \\
(1997)\end{array}$ & $\begin{array}{l}35 \text { niños obesos de } 7 \text { a } \\
11 \text { años }\end{array}$ & DXA & ECG & $\begin{array}{l}\text { En nińos obesos, el entrenamiento físico altera la función cardiaca } \\
\text { autónoma favorablemente, reduciendo el ratio de actividad } \\
\text { simpática-parasimpática. }\end{array}$ \\
\hline $\begin{array}{l}\text { Kaufman et al. } \\
(2007 \mathrm{a})\end{array}$ & $\begin{array}{l}36 \text { niños }(18 \text { đิ y } 18 \text { ○; } \\
11.5 \pm 0.8 \text { años })\end{array}$ & $\begin{array}{l}\text { IMC } \\
\text { DXA }\end{array}$ & ECG & $\begin{array}{l}\text { Niños obesos mayor ratio baja-alta frecuencia y menor actividad } \\
\text { del sistema nervioso parasimpático que los niños de peso normal. } \\
\text { Las relaciones entre estos factores parecen depender de la masa } \\
\text { grasa y/o de otros factores asociados con ser obeso. }\end{array}$ \\
\hline $\begin{array}{l}\text { Kaufman et al. } \\
\text { (2007b) }\end{array}$ & $\begin{array}{l}36 \text { niños (19 ̊̀ y } 17 \text { o; } \\
11.5 \pm 0.1 \text { años })\end{array}$ & $\begin{array}{l}\text { IMC } \\
\text { DXA }\end{array}$ & ECG & $\begin{array}{l}\text { Los niños con altos niveles de adiposidad tienen mayor proba- } \\
\text { bilidad de padecer disfunciones del sistema nervioso autónomo } \\
\text { cardiovascular y disfunción cardiovascular. }\end{array}$ \\
\hline $\begin{array}{l}\text { Martini et al. } \\
(2001)\end{array}$ & 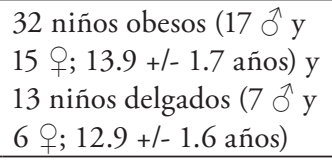 & IMC & ECG & $\begin{array}{l}\text { Los valores de VFC no fueron significativamente menores en los } \\
\text { niños obesos. } \\
\text { Aumento en la FC y en la TA asociado con un descenso en el } \\
\text { control parasimpático de la FC en los niños obesos. }\end{array}$ \\
\hline $\begin{array}{l}\text { Gutin et al. } \\
(2005)\end{array}$ & $\begin{array}{l}304 \text { adolescentes de } 14 \text { a } \\
18 \text { ańos }\end{array}$ & $\begin{array}{l}\text { IMC } \\
\text { Perímetro } \\
\text { Cintura } \\
\text { DXA }\end{array}$ & ECG & $\begin{array}{l}\text { Perfiles más favorables de VFC asociados con menos adiposidad } \\
\text { visceral y subcutánea. }\end{array}$ \\
\hline $\begin{array}{l}\text { Millis et al. } \\
\text { (2010) }\end{array}$ & $\begin{array}{l}\text { Adolescentes/jóvenes } \\
\text { sanos de sexo } \widehat{\overbrace{}}\end{array}$ & DXA & ECG & $\begin{array}{l}\text { Porcentaje de grasa corporal correlacionado negativamente con el } \\
\text { cociente baja frecuencia/alta frecuencia (LF/HF) durante la respi- } \\
\text { ración no controlada pero no durante la respiración rítmica. } \\
\text { Alto porcentaje de grasa corporal puede estar asociado con una } \\
\text { baja modulación simpática de la FC. }\end{array}$ \\
\hline
\end{tabular}


Tabla 2. Estudios destacados sobre la relación CC-VFC en adultos y mayores.

\begin{tabular}{|c|c|c|c|c|}
\hline Referencia & Muestra & $\begin{array}{l}\text { Medida de la } \\
\text { CC }\end{array}$ & $\begin{array}{l}\text { Medida de la } \\
\text { VFC }\end{array}$ & Relación CC/VFC \\
\hline $\begin{array}{l}\text { Christou et al. } \\
(2004)\end{array}$ & $\begin{array}{l}43 \text { đ: } 27 \text { jóvenes ( } 25 \\
\pm 1 \text { años) y } 16 \text { mayo- } \\
\text { res ( } 65 \pm 1 \text { años). }\end{array}$ & $\begin{array}{l}\text { DXA } \\
\text { IMC }\end{array}$ & ECG & $\begin{array}{l}\text { Mayor distribución de grasa corporal abdominal-perifé- } \\
\text { rica, mayor adiposidad abdominal y mayor adiposidad } \\
\text { total están asociadas con menores expresiones de VFC. }\end{array}$ \\
\hline $\begin{array}{l}\text { Esco et al. } \\
(2011)\end{array}$ & $\begin{array}{l}50 \lesssim(21.9 \pm 3.0 \\
\text { años) }\end{array}$ & $\begin{array}{l}\text { Pliegues de grasa } \\
\text { (pecho, abdomen } \\
\text { y muslo) } \\
\text { IMC y perímetro } \\
\text { Cintura }\end{array}$ & ECG & $\begin{array}{l}\text { Sumatorio de pliegues cutáneos (pecho, abdomen y mus- } \\
\text { lo) correlacionado significativamente con VFC. }\end{array}$ \\
\hline $\begin{array}{l}\text { Fagard et al. } \\
\text { (1999) }\end{array}$ & $\begin{array}{l}302 \text { ○े y } 312 \text { ㅇ }(25- \\
89 \text { años })\end{array}$ & IMC & ECG & $\begin{array}{l}\text { La influencia del tamańo corporal en la VFC es escasa e } \\
\text { insignificante. }\end{array}$ \\
\hline $\begin{array}{l}\text { Freeman et al. } \\
(1995)\end{array}$ & 597 ô & $\begin{array}{l}\text { IMC } \\
\text { Ratio cintura/ } \\
\text { cadera }\end{array}$ & ECG & $\begin{array}{l}\text { El peso y el IMC fueron predictores significativos de la } \\
\text { diferencia entre la frecuencia cardiaca máxima y mínima } \\
\text { (HRMax-Min). } \\
\text { El índice cintura-cadera y el porcentaje de grasa corporal } \\
\text { no fueron predictores significativos de las medidas de } \\
\text { VFC. } \\
\text { La VFC y el tamaño corporal están correlacionados. }\end{array}$ \\
\hline $\begin{array}{l}\text { Kim et al. } \\
(2005)\end{array}$ & $\begin{array}{l}19 \text { ๙ y } 22+(20-65 \\
\text { ańos) }\end{array}$ & $\begin{array}{l}\text { IMC } \\
\text { Ratio cintura/ } \\
\text { cadera } \\
\text { BIA }\end{array}$ & $\begin{array}{l}\text { Autonomic } \\
\text { Nervous System } \\
\text { Analyzer (SA- } \\
\text { 2000; Medicore, } \\
\text { Seoul, Korea) } \\
\end{array}$ & $\begin{array}{l}\text { RMSSD (que refleja la VFC) y LF (que refleja la } \\
\text { actividad simpática) se asociaron negativamente con el } \\
\text { porcentaje de masa grasa y con el ratio cintura/cadera en } \\
\text { personas obesas. }\end{array}$ \\
\hline $\begin{array}{l}\text { Martínez et al. } \\
\text { (2011) }\end{array}$ & $\begin{array}{l}110 \hat{\circlearrowright}(20.7 \pm 2.0 \\
\text { años) y } 118 \uparrow(20.2 \\
\pm 1.6 \text { años })\end{array}$ & $\begin{array}{l}\text { IMC } \\
\text { BIA }\end{array}$ & ECG & $\begin{array}{l}\text { Mayor tono simpático (LF) y VLF en hombres sanos con } \\
\text { un mayor porcentaje de músculo esquelético. } \\
\text { Mayor tono vagal (HF) y tono parasimpático (LF/HF) } \\
\text { en mujeres sanas con mayor porcentaje de grasa corporal. }\end{array}$ \\
\hline $\begin{array}{l}\text { Molfino et al. } \\
(2009)\end{array}$ & $\begin{array}{l}25 \text { adultos (13 ð̂ y } 12 \\
\text { 9) de } 20 \text { a } 60 \text { años }\end{array}$ & IMC & ECG & $\begin{array}{l}\text { Las HF (índice de la modulación parasimpática) en in- } \\
\text { dividuos con un IMC }<20 \mathrm{~kg} / \mathrm{m}^{2} \text { eran significativamente } \\
\text { más altas que las HF de los demás sujetos. }\end{array}$ \\
\hline $\begin{array}{l}\text { Murialdo et al. } \\
\text { (2007) }\end{array}$ & $\begin{array}{l}80 \text { + }(34 \text { con ano- } \\
\text { rexia, } 16 \text { con bulimia } \\
\text { nerviosa y } 30 \text { sanas), } \\
\text { entre } 14 \text { y } 45 \text { años }\end{array}$ & IMC & ECG & $\begin{array}{l}\text { Alteraciones del control simpático vagal de la VFC en } \\
\text { los desórdenes alimenticios. Estos cambios no estuvieron } \\
\text { relacionados con el peso corporal y el IMC. }\end{array}$ \\
\hline
\end{tabular}

Tabla 3. Correlaciones destacadas entre la CC y la VFC en niños y adolescentes

\begin{tabular}{lll}
\hline Referencia & Relación CC & Relación VFC \\
\hline $\begin{array}{l}\text { Kaufman } \text { et al. } \\
(2007 \mathrm{a})\end{array}$ & Masa Grasa & LF/HF y HFnu \\
\hline $\begin{array}{l}\text { Martini } \text { et al. } \\
(2001)\end{array}$ & Masa Grasa & $\begin{array}{l}\text { LF/HF, HF, Parámetros dimen- } \\
\text { sión de tiempo }\end{array}$ \\
\hline $\begin{array}{l}\text { Gutin } \text { et al. } \\
(2005)\end{array}$ & Masa Grasa & VFC \\
\hline $\begin{array}{l}\text { Millis } \text { et al. } \\
(2010)\end{array}$ & Masa Grasa & LF/HF \\
\hline \begin{tabular}{l}
${ }^{*}$ No se han encontrado relaciones significativas $)$ \\
\hline
\end{tabular}
\end{tabular}

( ${ }^{*}$ No se han encontrado relaciones significativas)
Tabla 4. Correlaciones destacadas entre la CC y la VFC en adultos y mayores.

\begin{tabular}{lll}
\hline Referencia & Relación CC & Relación VFC \\
\hline Christou et al. (2004) & Masa Grasa & VFC \\
\hline Esco et al. (2011) & Masa Grasa & VFC \\
\hline Fagard et al. (1999) & Tamańo corporat* & VFC* \\
\hline Freeman et al. (1995) & El peso y el IMC & HRMax-Min \\
\cline { 2 - 3 } & Masa Grasa* & VFC* \\
\cline { 2 - 3 } & Tamańo corporal & VFC \\
\hline Kim et al. (2005) & Masa Grasa & LF y RMSSD \\
\hline Martínez et al. (2011) & Masa Muscular & LF y VLF \\
\cline { 2 - 3 } & Masa Grasa & LF/HF y HF \\
\hline Molfino et al. (2009) & IMC & HF \\
\hline
\end{tabular}




\subsection{En relación a la obesidad}

En los apartados anteriores hemos analizado la influencia de la composición corporal en la variabilidad de la frecuencia cardiaca en nińos, adolescentes, adultos y mayores. Ha quedado clara la existencia de una relación entre la VFC y la composición corporal, lo que hace pensar que la obesidad puede estar directamente relacionada con la VFC.

En esta línea, Kim et al. (2005) han observado una asociación negativa entre el RMSSD (que refleja la variabilidad de la frecuencia cardiaca) y LF (que refleja la actividad simpática) con el porcentaje de masa grasa y con el ratio cintura/ cadera en personas obesas. Por tanto, se plantea la hipótesis de que la obesidad puede cambiar la respuesta cardiaca nerviosa autónoma, lo que explicaría, al menos parcialmente, el mecanismo por el cual la obesidad aumenta la mortalidad cardiaca (Kim et al., 2005).

Algunos autores han observado que los niños obesos tienen un ratio baja-alta frecuencia significativamente elevado y una medida de la actividad del sistema nervioso parasimpático significativamente baja con respecto a los niños de peso normal. Siguiendo esta línea de argumentación, se ha observado que dicha población se caracteriza por presentar una disfunción cardiovascular autónoma y un aumento de la leptina, la resistencia a la insulina, el estrés oxidativo y la inflamación. Las relaciones entre estos factores parecen estar estrechamente relacionadas con la cantidad de masa, aunque no se pueden descartar otros factores asociados con dicha patología (Kaufman et al., 2007a). Esto ha desembocado en que varios autores consideran que los niños con altos niveles de adiposidad tienen mayor probabilidad de padecer disfunciones del sistema nervioso autónomo cardiovascular y disfunción cardiovascular (Kaufman et al., 2007b). Además, parece ser que los niños obesos tienen una mayor frecuencia cardiaca, una mayor tensión arterial y un menor control parasimpático sobre los ritmos del corazón (Martini et al., 2001).

En definitiva, se puede afirmar que los sujetos con obesidad tienen en general una frecuencia cardiaca más rápida y una disminución de la variabilidad de la frecuencia cardiaca. Debido a las anormalidades en el balance simpático-vagal que se registran en sujetos obesos, se ha determinado que se pueden considerar como factores relacionados con el aumento del riesgo de muerte súbita (Lavie et al., 2009; LópezJiménez \& Cortés-Bergoderi, 2011).

\subsection{En relación al sexo}

En relación al sexo, no muchos trabajos de investigación analizan la relación entre composición corporal y variabilidad de la frecuencia cardiaca en función del sexo. Al respecto, podemos destacar las aportaciones de Ramaekers et al. (1998), que señalan que la modulación autónoma cardiaca determinada por la VFC es significativamente más baja en mujeres sanas que en hombres sanos. Esto puede ser debido a una actividad simpática más baja en las mujeres, lo que puede protegerlas contra arritmias y contra el desarrollo de enfermedades coronarias del corazón.

Cuando las mujeres son jóvenes tienen una modulación parasimpática más alta y una modulación simpática más baja que los hombres, pero las diferencias van desapareciendo a lo largo de la vida. Esto puede ser debido al rol de los estrógenos, y podría ayudar a explicar la protección contra la enfermedad cardiovascular en las mujeres más jóvenes y la pérdida de esta protección tras la menopausia (Fagard et al., 1999).

Sin embargo, Gutin et al. (2005) no encuentran diferencias en la RMSSD de la VFC, cuando son estudiados adolescentes de distinto sexo. Al igual que en el estudio anterior, Henje Blom et al. (2009) no reportan diferencias significativas en los parámetros de la variabilidad de la frecuencia cardiaca (HF, LF o SDNN) en función del género. En contraposición, Silvetti et al. (2001) han encontrado valores significativamente más elevados en la desviación estándar del intervalo RR (SDNN) en chicos, cuando estos son comparados con un grupo de características similares de género femenino. Sin embargo, en lo que sí coinciden la mayor parte de estos estudios, es en que los chicos tienen una menor frecuencia cardiaca que las chicas.

\subsection{En relación a la cantidad de actividad física realizada}

Algunos autores han encontrado también relaciones entre la actividad física realizada por las personas, su composición corporal y su variabilidad de la frecuencia cardiaca. Se ha encontrado que los perfiles más favorables de variabilidad de la frecuencia cardiaca están asociados con más actividad física moderada-vigorosa, con mejor condición física cardiovascular y con menos adiposidad visceral y subcutánea. La mejora de la modulación cardiaca autónoma puede ser una vía a través de la cual la actividad física, la condición física y la delgadez contribuyen a la salud cardiovascular desde edades tempranas (Gutin et al., 2005).

Henje Blom et al. (2009) encontraron correlaciones significativas entre la actividad física realizada, la frecuencia cardiaca y algunos parámetros de VFC (HF, LF, SDNN) en adolescentes sanos. En la misma línea, en adultos, el ejercicio aeróbico regular resulta en una reducción de la frecuencia cardiaca, lo que refleja un aumento de la influencia vagal en el ritmo cardiaco (Hottenrott et al. 2006). El ejercicio físico de intensidad baja-moderada se asocia con cambios favorables en la VFC en hombres adultos (Tuomainen et al., 2005; Fagard et al., 1999). Y en lo que se refiere a los efectos del entrenamiento, parece tener una influencia limitada en la VFC cuando es inferior a siete semanas (Buchheit \& Gindre 2006; Gamelin et al. 2009). 


\section{Conclusiones}

\section{Conclusión general:}

Además de la relación que estos dos parámetros (CC y VFC) tienen de forma independiente con el estado de salud de las personas, esta revisión, permite concluir que también existe una relación entre la composición corporal y la variabilidad de la frecuencia cardiaca. Aunque todavía existen algunas discrepancias acerca de qué elementos de la composición corporal y de la variabilidad de la frecuencia cardiaca se correlacionan en mayor medida, lo que si parece estar claro es que una disminuida VFC está asociada a un alto porcentaje de grasa corporal y a un menor porcentaje de masa muscular. Esto hace pensar que una reducción del porcentaje de grasa corporal y un aumento de la masa muscular podría ser un tratamiento eficaz para mejorar la VFC.

\section{Conclusiones específicas:}

1. La variabilidad de la frecuencia cardiaca es un parámetro muy útil a la hora de determinar el estado de salud de diferentes grupos poblacionales, por lo que su estudio tiene una gran aplicación en el ámbito de las ciencias de la actividad física y del deporte.

2. La determinación de la composición corporal es fundamental para prevenir la aparición de patologías relacionadas con un alto porcentaje de masa grasa, tales como la obesidad, diabetes tipo II y enfermedades cardiovasculares.

3. La composición corporal y la variabilidad de la frecuencia cardiaca están estrechamente relacionadas, lo cual resulta de suma relevancia a la hora de realizar un diagnóstico sobre estado de salud, así como para prevenir posibles enfermedades.

4. En función de la edad, se observa que tanto en niños como en adolescentes, un mayor porcentaje de masa grasa parece estar asociado a una disminución de la variabilidad de la frecuencia cardiaca.

5. En relación a la obesidad, se puede concluir que las personas obesas muestran valores menos favorables de variabilidad de la frecuencia cardiaca, aumentando el riesgo de aparición de enfermedades asociadas a dicha patología.

6. Los resultados observados en relación al sexo, parecen evidenciar una menor actividad simpática en mujeres que en los hombres, factor que podría estar relacionado con la cardioprotección premenopáusica por influencia estrogénica.

7. En relación a la cantidad de actividad física realizada, está perfectamente demostrada la influencia positiva que ejerce la actividad física sobre los parámetros tanto de composición corporal como de la variabilidad de la frecuencia cardiaca.

\section{Futuras líneas de investigación}

En primer lugar es conveniente que se lleven a cabo más estudios para determinar la validez y la fiabilidad de los diferentes métodos de evaluación de la composición corporal y de la variabilidad de la frecuencia cardiaca, a fin de conocer cuáles son los ideales en función del ámbito específico de aplicación: medicina deportiva, entrenamiento, educación física escolar, etc.

En segundo lugar, sería muy interesante el diseño y aplicación de programas de intervención específicos (fuerza, resistencia, etc.) para conocer el efecto sobre los distintos parámetros de la CC y VFC en diferentes grupos poblacionales (obesos, cardiópatas, $3^{\text {a }}$ edad, etc.).

En tercer lugar, esta revisión ha puesto de manifiesto que hasta el momento no se han realizado estudios descriptivos sobre el comportamiento de estas dos variables (VFC y CC) en la franja de edad comprendida entre los 0 y los 6 ańos. Por lo tanto, sería interesante aportar datos al respecto.

Por último, a pesar de ser demasiado ambicioso, la realización de un estudio epidemiológico que permita relacionar aspectos como la alimentación, la actividad física, la VFC y la composición corporal, aportaría mucha y muy valiosa información para el diseño de estrategias de prevención de enfermedades relacionadas con los hábitos de vida (poco saludables) que se están instaurando en la actual sociedad. Además, de esta forma, se podrían extraer normogramas en función de diferentes parámetros, como por ejemplo la edad, el género, etc.

En definitiva, la revisión bibliográfica realizada en este trabajo pretende abrir camino a posteriores estudios y trabajos más específicos sobre la relación entre la composición corporal y la variabilidad de la frecuencia cardiaca y su influencia en el estado de salud de las personas.

\section{Bibliografía}

1. Achten, J. \& Jeukendrup, E. (2003). Heart rate monitoring: applications and limitations. Sports Medicine, 33(7), 517-538.

2. Alburquerque Sendín, F. (2008). Estudio comparativo intermetodológico de la composición corporal (Antropometría, BIA y DEXA). Tesis doctoral. Universidad de Salamanca.
3. Alburquerque Sendín, F., Martín-Vallejo, F. J., García Talavera, P., Martín Gómez, M. E. \& Santos del Rey, M. (2010). Comparison of fat mass and fat-free mass between Anthropometry, BIA and DEXA in young females: are methods really interchangeable? European Journal of Anatomy, 14(3), 133-141. 
4. Alvero, J. R., Diego, A. M., Fernández, V.J. \& García, J. (2004). Métodos de evaluación de la composición corporal: Tendencias actuales I. Archivos de Medicina del Deporte, 21(104), 535-538.

5. Alvero, J. R., Diego, A. M., Fernández, V.J. \& García, J. (2005a). Métodos de evaluación de la composición corporal: Tendencias actuales II. Archivos de Medicina del Deporte, 22(105), 45-49.

6. Alvero, J. R., Diego, A. M., Fernández, V.J. \& García, J. (2005b). Métodos de evaluación de la composición corporal: Tendencias actuales III. Archivos de Medicina del Deporte, 22(106), 121-127.

7. Alvero, J. R., Cabañas, M. D., Herrero, A., Martínez, L., Moreno, C., Porta, J., Sillero, M. \& Sirvent, J. E. (2010). Protocolo de valoración de la composición corporal para el reconocimiento médico-deportivo. Documento de consenso del Grupo Español de Cineantropometría (GREC) de la Federación Española de Medicina del Deporte (FEMEDE). Versión 2010. Archivos de Medicina del Deporte, 27(139), 330-344.

8. Björntorp, P. (1992). Male fat distribution and cardiovascular risk. Blood Pressure, 1(suppl. 4), 17-19.

9. Buchheit, M. \& Gindre, C. (2006). Cardiac parasympathetic regulation: respective associations with cardiorespiratory Fitness and training load. American Journal of Physiology Heart and Circulatory Physiology, 291, H451-H458

10. Christou, Jones, Pimentel \& Seals (2004). Increased abdominal-toperipheral fat distribution contributes to altered autonomic-circulatory control with human aging. American Journal of Physiology - Heart and Circulatory Physiology, 287(4), H1530-H1537.

11. De Becker, P., Dendale, P., De Meirleir, K., Campine, L., Vandenbme, K. \& Hagers, Y. (1998). Autonomic testing in patients with chronic fatigue syndrome. American Journal of Medicine, 105, 22S-26S.

12. Esco, M. R., Williford, H. N. \& Olson, M. S. (2011). Skinfold thickness is related to cardiovascular autonomic control as assessed by heart rate variability and heart rate recovery. Journal of Strength and Conditioning Research, 25(8), 2304-2310.

13. Fagard, R. H., Pardaens, K. \& Staessen, J. A. (1999). Influence of demographic, anthropometric and lifestyle characteristics on heart rate and its variability in the population. Journal of Hypertension, 17(11), 1589-1599.

14. Freeman, Weiss, Roberts, Zbikowski \& Sparrow (1995). The relationship between heart rate variability and measures of body habitus. Clinical Autonomic Research, 5(5):261-266.

15. Gallo, J., Farbiarz, J. \& Álvarez, D. (1999). Análisis espectral de la variabilidad de la frecuencia cardíaca. Iatreia, 12(2), 61-71.

16. Gamelin, F. X., Berthoin, S. \& Bosquet, L. (2006). Validity of the Polar s810i heart rate monitor to measure RR intervals at rest. Medicine \& Science in Sports \& Exercise, 38(5), 887-893.

17. Gamelin, F. X., Baquet, G., Berthoin, S., Thevenet, D., Nourry, C., Nottin, S. \& Bosquet, L. (2009). Effect of high intensity intermittent training on heart rate variability in prepubescent children. European Journal of Applied Physiology, 105, 731-738.

18. Grund, A., Krause, H. \& Kraus, M., Siewers, M., Rieckert, H. \& Müller, M. J. (2001). Association between different attributes of physical activity and fat mass in untrained, endurance and resistance trained men. European Journal of Applied Physiology, 84(4), 310-320.

19. Gutin, B., Owens, S., Slavens, G., Riggs, S. \& Treiber, F. (1997). Effect of physical training heart-period variability in on obese children. Journal of Pediatrics, 130 (6), 938-943.

20. Gutin, B., Howe, C., Johnson, M. H., Humphries, M. C., Snieder, H. \& Barbeau, P. (2005). Heart Rate Variability in Adolescents: Relations to Physical Activity, Fitness, and Adiposity. Medicine \& Science in Sports \& Exercise, 37(11), 1856-1863.

21. Gutin, B. \& Owens, S. (2011). The Influence of Physical Activity on Cardiometabolic Biomarkers in Youths: A Review. Pediatric Exercise Science, 23(2), 169-185.

22. Hall, M., Vasko, R., Buysse, D., Ombao, H., Chen, Q., Cashmere, D.,
Kupfer, D. \& Thayer, J. (2004). Acute Stress Affects Heart Rate Variability During Sleep. Psychosomatic Medicine, 66, 56-62.

23. Henje Blom, E., Olsson, E. M. G., Serlachius, E., Ericson, M. \& Ingvar, M. (2009). Heart rate variability is related to self-reported physical activity in a healthy adolescent population. European Journal of Applied Physiology, 106, 877-883.

24. Hynynen, E., Uusitalo, A., Konttinen, N. \& Rusko, H. (2006). Heart rate variability during night sleep and after awakening in overtrained athletes. Medicine \& Science in Sports \& Exercise, 38(2), 313-317.

25. Hottenrott, K., Hoos, O. \& Esperer, H. D. (2006). Heart rate variability and physical exercise. Current status. Herz, 31, 544-552.

26. Kaufman, C. L., Kaiser, D. R., Steinberger, J., Kelly, A. S. \& Dengel, D. R. (2007a). Relationships of Cardiac Autonomic Function With Metabolic Abnormalities in Childhood Obesity. Obesity, 15(5), 1164-1171.

27. Kaufman, C. L., Kaiser, D. R., Steinberger, J. \& Dengel, D. R. (2007b) Relationships between heart rate variability, vascular function, and adiposity in children. Clinical Autonomic Research, 17(3), 165-171.

28. Kim, J. A., Park, Y. G., Cho, K. H., Hong, M. H., Han, H. C., Choi, Y. S. \& Yoon, D. (2005). Heart Rate Variability and Obesity Indices: Emphasis on the Response to Noise and Standing. Journal of the American Board of Family Practice, 18(2), 97-103.

29. Kleiger, R. E., Bosner, M. S., Rottmans, J. N. \& Stein, P. K. (1992). Time domain measurements of heart rate variability. Cardiology Clinics,10(3), 487-498.

30. Kleiger, R., Stein P., Thomas Bigger, Jr. J. (2005). Heart rate variability: Measurement and clinical utility. Annals of Noninvasive Electrocardio$\log y, 10(1), 88-101$.

31. Kop, W. J., Verdino, R. J., Gottdiener, J. S., O’Leary, S. T., Bairey Merz, C. N. \& Krantz, D. S. (2001). Changes in heart rate and heart rate variability before ambulatory ischemic events. Journal of the American College of Cardiology, 38,742-749.

32. Lavie, C. J., Milani, R. V. \& Ventura, H. O. (2009). Obesity and cardiovascular disease: risk factor, paradox, and impact of weight loss. Journal of the American College of Cardiology, 53, 1925-1932.

33. López-Jiménez, F. \& Cortés-Bergoderi, M. (2011). Obesidad y corazón. Revista Española de Cardiología, 64(2), 140-149.

34. Malpas, S. C. \& Maling, T. J. (1990). Heart rate variability and cardiac autonomic function in diabetes. Diabetes, 39(10), 1177-1181.

35. Martinez, D., Rebolledo, M. E., González, E., Duncker, D. M. \& Duncker, M. E.M. (2011). Sympathovagal Tone Assessed by Heart Rate Variability is Directly Related to Body Mass Index, Percent Body Fat, and Skeletal Muscle in Healthy Male and Female Young Volunteers. Cleveland Clinic Journal of Medicine, 78(S1), S94.

36. Martinez-Lavin, M. (2002). The autonomic nervous system and fibromyalgia. Journal of musculoskeletal pain, 10, 221-228.

37. Martini, Riva, Rabbia, Molini, Ferrero, Cerutti, Carra \& Veglio (2001). Heart rate variability in childhood obesity. Clinical Autonomic Research, 11(2), 87-91.

38. Maud P. J. \& Foster C. (2006). Physiological assessment of human fitnessSecond Edition. Champaign, Illinois: Human Kinetics.

39. Millis, Austin, Hatcher, Bond, Faruque, Goring, Hickey \& DeMeersman (2010). Association of body fat percentage and heart rate variability measures of sympathovagal balance. Life Sciences, 86(5-6), 153-157.

40. Molfino, A., Fiorentini, A., Tubani, L., Martuscelli, M., Fanelli F.R. \& Laviano, A. (2009). Body mass index is related to autonomic nervous system activity as measured by heart rate variability. European Journal of Clinical Nutrition, 63(10), 1263-1265.

41. Moreno Villares, J. M. (2000). Técnicas de valoración de la composición corporal. Actas del XXIX Congreso Nacional Ordinario de Pediatría de la Asociación Española de Pediatría.

42. Murialdo, G., Casu, M., Falchero, M., Brugnolo, A., Patrone, V., Cerro, P. F., Ameri, P., Andraghetti, G., Briatore, L., Copello, F., Cordera, R., Rodriguez, G., Ferro, A. M. (2007). Alterations in the autonomic 
control of heart rate variability in patients with anorexia or bulimia nervosa: Correlations between sympathovagal activity, clinical features, and leptin levels. Journal of Endocrinological Investigation, 30(5), 356362.

43. Oria, E., Lafita, J., Petrina, E. \& Argüelles, I. (2002). Composición corporal y obesidad. Body composition and obesity. Anales del Sistema Sanitario de Navarra, 25(1), 91-102.

44. Pumprla, J., Howorka, K., Groves, D., Chester, M. \& Nolan, J. (2002) Functional assessment of heart rate variability: physiological basis and practical applications. International Journal of Cardiology, 84(1), 1-14.

45. Ramaekers, D., Ector, H., Aubert, A. E., Rubens, A., Van de Werf, F. (1998). Heart rate variability and heart rate in healthy volunteers. European Heart Journal, 19, 1334-1341.

46. Rodas, G., Pedret, C., Ramos, J. \& Capdevila, L. (2008a). Variabilidad de la Frecuencia Cardiaca: concepto, medidas y relación con aspectos clínicos I. Archivos de Medicina del Deporte, 25(123), 41-47.

47. Rodas, G., Pedret, C., Ramos, J. \& Capdevila, L. (2008b). Variabilidad de la Frecuencia Cardiaca: concepto, medidas y relación con aspectos clínicos II. Archivos de Medicina del Deporte, 25(124), 119-127.

48. Sibolboro, E., Kelsey, R., Katkin, E. \& Sloan, R. (2001). Vagal rebound and recovery from psychological stress. Psychosomatic medicine, 63, 650-657.

49. Silvetti, M. S., Drago, F. \& Ragonese, P. (2001). Heart rate variability in healthy children and adolescents is partially related to age and gender. International Journal of Cardiology, 81, 169-174.

50. Task Force of the European Society of Cardiology and the North American Society of pacing and electrophysiology (1996). Heart Rate Variability. Standards of Measurement, Physiological Interpretation, and Clinical Use. Circulation, 93, 1043-1065.

51. Task Force of the European Society of Cardiology and the North American Society of pacing and electrophysiology (1996). Heart Rate Variability. Standards of Measurement, Physiological Interpretation, and Clinical Use. European Heart Journal, 17, 354-381.

52. Tuomainen, P., Peuhkurinen, K., Kettunen, R. \& Rauramaa, R. (2005). Regular physical exercise, heart rate variability and turbulence in a 6-year randomized controlled trial in middle-aged men: the DNASCO study. Life Sciences, 77, 2723-2734.

53. Zahorska-Markiewicz, B., Kuagowska, E., Kucio, C., Klin, M. (1993) Heart rate variability in obesity. International journal of obesity and related metabolic disorders, 7(1), 21-23.

\section{Abreviaturas utilizadas}

BIA: Bioimpedancia eléctrica.

$C C$ : Composición Corporal.

$D X A$ (DEXA): Absorciometría de Rayos $\mathrm{X}$ de Energía Dual.

ECG: Electrocardiograma.

E/I ratio: Cociente expiración/inspiración.

$F C$ : Frecuencia cardiaca.

$H F$ : Alta frecuencia (entre 0,15 y $0,4 \mathrm{~Hz}$ ).

HFnu: Unidades normalizadas de alta frecuencia.

HRMax-Min: Diferencia entre la frecuencia cardiaca máxima y mínima.

$H R V$ : Heart Rate Variability (Variabilidad de la Frecuencia Cardiaca).

IMC: Índice de Masa Corporal.

$L F$ : Baja frecuencia (entre 0,04 y 0,15 Hz).
LF/ HF: Proporción entre las bajas frecuencias y las altas frecuencias.

$M G$ : Masa Grasa.

$M L G$ : Masa Libre de Grasa.

RMSSD ( $r$-MSSD, $r M S S D)$ : Raíz cuadrada del valor medio de la suma de las diferencias al cuadrado de todos los intervalos RR sucesivos.

$R R(N N)$ : Intervalo entre dos latidos.

$S N A$ : Sistema nervioso autónomo.

SNP: Sistema nervioso parasimpático.

SNS: Sistema nervioso simpático.

TA: Tensión arterial.

VFC: Variabilidad de la Frecuencia Cardiaca.

$V L F$ : Muy baja frecuencia $(0,003$ a $0,04 \mathrm{~Hz})$.

Financiación: Fundación Séneca - Agencia de Ciencia y Tecnología de la Región de Murcia. Consejería de Industria, Turismo, Empresa e Innovación de la CARM. 